\title{
A focused ethnography of how endoscopy practitioners utilize capnography in sedated patients
}

\author{
Deemah Aldossary, ${ }^{1}$ Sherran Milton ${ }^{2}$ \\ ${ }^{1}$ Department of Anesthesia Technology, Prince Sultan Military College of Heath Sciences, Dhahran, Saudi Arabia; ${ }^{2}$ School of Healthcare \\ Sciences, Cardiff University, Cardiff, United Kingdom
}

\begin{abstract}
The literature shows that respiratory complications are common with sedation. Given the inherent risk, capnography monitoring is recommended whenever sedation is administered. We aimed to explore sedation practitioners' behavior patterns and perceptions regarding capnography monitoring during endoscopy sedation and examine how capnography influenced clinical decision making when assessing respiration. We conducted a focused ethnography with triangulated observations and semi-structured interviews, and we purposively sampled and recruited five sedation practitioners as participants at a hospital in Saudi Arabia. Through data analysis, we identified representative themes and found cultural differences between anesthesia practitioners and nurses when using capnography during sedation. Anesthesia practitioners linked safety and the use of capnography to maintain adequate respiration, while nurses believed capnography was a secondary supportive monitor to patient observation and assessment. Findings also captured the unique cultures and values of each professional group to be associated with the varying perceptions. We also identified several factors facilitating and barring adequate utilization of capnography. In conclusion, professional culture, hospital policy, knowledge and previous experience with capnography guided the monitoring practice of endoscopy practitioners when assessing respiration during sedation.
\end{abstract}

\section{Introduction}

The last decade has witnessed considerable growth in complications resulting from over-sedation during endoscopy, with respiratory depression and subsequent hy-

Correspondence: Deemah Nassir Aldossary, Department of Anesthesia Technology, Prince Sultan Military College of Heath Sciences, Dhahran 34313, Saudi Arabia.

Tel.: +966 544807995 .

Fax: +966 138440000 .

E-mail: deemah@psmchs.edu.sa

Key words: End-tidal Co2; ventilation; perception; patient safety.

Funding: This work was supported by the Saudi Arabian Cultural Bureau in London.

Conflict of interest: The authors declare no potential conflict of interests.

Acknowledgements: The authors would like to thank Dr. Mohammad Albeshir, who helped us to enter the field and recruit participants.

Received for publication: 3 May 2020.

Accepted for publication: 14 September 2020.

This work is licensed under a Creative Commons Attribution NonCommercial 4.0 License (CC BY-NC 4.0).

${ }^{\circ}$ Copyright: the Author(s), 2020

Licensee PAGEPress, Italy

Qualitative Research in Medicine \& Healthcare 2020; 4:83-93

doi:10.4081/qrmh.2020.9077 poxemia the most commonly reported. ${ }^{1,2}$ In a report from the Joint Commission in 2017 about the standards for hospital requirements, ${ }^{3}$ inadequate monitoring during sedation accounted for $58 \%$ of cases of respiratory depression. Given the inherent risk with sedation, the European Board of Anesthesiology in 2011 and the American Society of Anesthesiology $y^{5}$ in 2010 updated their recommendations and emphasized that ventilation must be monitored via capnography during moderate and deep sedation outside the operating theatre. Capnography is a sensitive monitor that works as an early warning system of respiratory depression and apnea before the development of subsequent hypoxemia. ${ }^{2}$ Adequate interpretation of capnography requires practitioners to have a thorough understanding of monitoring parameters and the technical aspects of capnography equipment. ${ }^{6}$

The consensus in the literature is that capnography monitoring has great value for patient safety and adequate ventilation during endoscopy sedation. ${ }^{7-10}$ However, recommendations for the routine use of capnography during sedation have raised questions about the burden of false alarms and the cost of purchasing equipment and training practitioners. ${ }^{8}$ The literature regards the skills of the monitoring practitioner as essential for the adequate interpretation of capnography parameters. ${ }^{9}$ However, less is known about how capnography might influence staff behavior, and how they use this advanced technology to assess ventilation during sedation.

$\operatorname{Egan}^{10}$ argued that introducing and implementing capnography successfully in the endoscopy unit provides practitioners the ability to monitor patients' ventilation under sedation with greater confidence. However, Lang- 
han et al. ${ }^{11}$ reported that capnography was underutilized for sedation outside the operating theatre despite its availability and updated standards supporting its use. Further, Langhan suggested that practitioners were unfamiliar with capnography, leading to poor utilization patterns. In this focused ethnographic study, we aim to explore sedation practitioners' behavior and perceptions towards capnography during endoscopy sedation; and how capnography monitoring influences clinical decision-making while assessing ventilation during sedation. We will also identify the factors facilitating and barring the use of capnography.

\section{Materials and Methods}

\section{Design}

In this qualitative research, we used a focused ethnographic design, a suitable pragmatic approach to capture specific cultural perspectives of the practice of capnography monitoring during endoscopy sedation. Brewer ${ }^{12}$ has defined ethnography as the study of people within their cultural context to explore and reveal their behavior, beliefs, knowledge, and shared social meanings. A focused ethnography typically addresses a specific shared experience within a sub-cultural group rather than the entire community. ${ }^{13}$ Within this focused ethnography, practitioners shared the specific feature of using capnography monitoring during sedation. We formulated pre-determined aspects of inquiry before entering the field, and we limited the observation to a short period due to the nature of focused ethnography. ${ }^{13}$ Ethnographic findings describe individuals within a holistic perspective and generate a basis for future cultural changes in practice.

\section{Sample and setting}

We conducted the study in the endoscopy unit of a large regional hospital in the eastern province of Saudi Arabia. A focused ethnography usually involves a limited number of participants, ${ }^{14}$ and we used purposive sampling to recruit key individuals as participants. According to Patton, ${ }^{15}$ purposive sampling in qualitative research facilitates access to key informants who have knowledge of and experience with the phenomenon of interest, which is suitable for this focused ethnography. The sample consisted of sedation practitioners: anesthetists, anesthesia technicians, and endoscopy nurses. The director of the research center at the hospital introduced us to a gatekeeper (someone who has access to the field), and we worked closely with him to choose a list of potential participants. The inclusion criteria were as follows: individuals who were an anesthetist, anesthesia technician, or endoscopy nurse; working in the endoscopy unit; involved with patient monitoring during sedation; had more than three years' experience working in endoscopy sedation; and agreed to take part in the study. We initially approached seven participants, and five agreed to take part in the study. The final sample consisted of two anesthetists, one anesthesia technician, and two sedation nurses.

\section{Entrance to the field and recruitment}

We gained access to the site after obtaining ethical approval from the hospital. The gatekeeper arranged entrance to the endoscopy unit and recruitment of key participants.

\section{Data collection}

We conducted the study in two phases. In the first phase, we performed non-participant observation to explore the practice of capnography monitoring during sedation; and in the second phase, we carried out semi-structured interviews of the same study participants were observed.

\section{Observations and field notes}

Observation allows an exploration of culture by capturing behavior patterns in a continual and dynamic manner. ${ }^{12}$ We deemed the non-participant observation as a suitable approach for this focused ethnography. ${ }^{16}$ Nonetheless, collecting cultural data requires some degree of participant observation to enable researchers to experience the lives of participants under study ${ }^{17}$ However, we could not perform participant observation based on the ethical approval, which limited our involvement to observe and not interfere with practice. We took every opportunity to spend time in the field to observe the practice of capnography monitoring during sedation within a predetermined timeframe, from 25 November 2018 through 30 December 2018. We had limited time in the field; thus, we regarded focused ethnography as appropriate ${ }^{14}$ to obtain practitioners' perceptions of the use of capnography monitoring during sedation.

We had a genuine concern in the field to not disturb practitioners as they were performing their duties, so we maintained distance from participants as they were administering sedation and approached participants only as circumstances allowed. For example, we maintained distance during the induction of/recovery from sedation to avoid distracting the sedation team.

We did not observe participants in a particular order, but based on the availability of practitioners and the number of scheduled cases from 8 am through 2 pm. Endoscopy cases took place three days per week, with seven to nine cases performed each day. The more sophisticated and combined procedures (e.g. esophago-gastro-duodenoscopy and colonoscopy) took place in the morning, when anesthetists performed deep sedation. The endoscopy unit was quieter in the afternoon, when generally nurses administered moderate sedation under the supervision of the endoscopy physician, mostly for colonoscopies. Each observation session lasted 20-50 minutes, depending on the procedure. 
The overall context of the observation was fundamental to the quality of the data we collected. ${ }^{17}$ The process of describing what we observed led to focused observation of participants while using capnography. We included in our field notes descriptive details of the people, place, noise, location of monitoring equipment in the room, and so on. Were recorded the field notes by hand with the aid of an observational checklist we constructed and validated by two anesthesia consultants for appropriateness and clarity. Observational checklists allow researchers to focus on recording what is relevant to the study objectives. ${ }^{17} \mathrm{We}$ indicated the date, time, pseudonym of the participant, and the duration of the observation period in each checklist and field note. We maintained close proximity to participants and focused on recording how participants used capnography, how they responded to alarms, how they interacted around capnography whenever there was an alarm, and how and when they initiated an intervention based on capnography alarms. During observations, we only jotted short notes; we accessed a private room to write full descriptions at the end of each observation session.

\section{Semi-structured interviews}

We adopted the interview approach from Bryman. ${ }^{18}$ We relied on a pre-determined interview guide, consisting of standard questions to allow data comparison among participants. However, we asked additional questions when needed to expand upon certain points that emerged in a reflexive manner during observations. We started each interview by collecting demographic information, followed by asking relatively straightforward, factual questions such as "how many years have you been practicing sedation?", to help establish rapport with the participant. We used probes to confirm the data we collected were relevant to the objectives of the study.

We discussed four main themes in the interviews: sedation practice and patient safety culture, sedation complications and subsequent interventions, capnography monitoring and performance, and factors facilitating and barring the use of capnography. We drew these themes from the literature and discussed them repeatedly to ensure they were credible and representative. We interviewed participants individually for approximately $15-20$ minutes in a private room in the endoscopy unit after completion of observation sessions. We audio recorded interviews and transcribed them verbatim.

\section{Triangulation}

Collecting data from a range of sources is known as data triangulation. ${ }^{14}$ This approach allowed us to explore the phenomenon from multiple perspectives to meet the research objectives. ${ }^{19} \mathrm{We}$ combined the findings from observation and interviews to produce a comprehensive picture of the phenomenon that neither approach could provide alone. Triangulating data maintains rigor and increases the credibility of the findings of qualitative research. ${ }^{20}$

\section{Ethical consideration}

This study was part of a master's student project at Cardiff University. The study commenced on $25^{\text {th }}$ November 2018 after obtaining the necessary ethical approvals by the ethics committee of Cardiff University School of Healthcare Sciences and by the regional hospital in Saudi Arabia. We sent an information sheet to all participants, and obtained a signed written consent before beginning the study. We adhered to the guidelines of Cardiff University ${ }^{21}$ and the Data Protection $\mathrm{Act}^{22}$ when collecting and holding data. In order to ensure the anonymity of participants, we assigned them pseudonyms to protect their identities.

\section{Analysis}

\section{Observational data analysis}

We initiated analysis by repeatedly reading the descriptive field-notes to understand the experiences of participants and compare the different layers of data with one another. We then re-read the data to generate initial codes. Based on Ryan and Bernard' ${ }^{23}$ method of coding, we grouped codes according to similarity to generate representative themes. We repeatedly broke down, reassembled, and compared themes to provide an explanation, and once no more information emerged, we then addressed the next concept.

\section{Analysis of semi-structured interviews}

We analyzed the interviews following guidance by Steinar Kvale ${ }^{24}$ for interviews in-depth analysis. We used one of Kvale's modes of interview analysis: Analyzes focusing on meaning, as summarized in Table 1.

\section{Rigor}

Fieldwork requires a degree of flexibility and adaptability to overcome challenges. ${ }^{17}$ We maintained a reflexive diary to remain objective in the field and avoid letting personal interpretations to obscure the participants' reality. To ensure the trustworthiness of the study,$^{20}$ we recorded emotions, feelings, thoughts, and impressions as they occurred. We also maintained an audit trail to support a clear transition from coding to final themes. An audit trail describes how we transparently collected and analyzed data in a logical way, ${ }^{25}$ and includes examples of the coding process and descriptions of the grouping of codes into relevant, meaningful themes. To minimize bias, we analyzed all data together after completing the data collection.

\section{Results}

Five sedation practitioners agreed to take part in this study. We observed participants when monitoring their sedated patients, followed up by interviews. The participants were two anesthetists, one anesthesia technician, and two sedation nurses. Table 2 provides a systematic description 
of the observations based on Tattersall's Five W's approach $^{26}$ to systematically describe a situation. Table 3 presents a summary of participants' characteristics. Data analysis revealed representative themes, and we grouped themes four main categories: safety culture of endoscopy sedation, professional identity and role in safe sedation practice, personal experience and knowledge about capnography performance, and facilitators and barriers to the utilization of capnography. We present themes and illustrative quotes below, triangulated with the observations.

Table 1. Summary of Kvale's steps for interviews in-depth analysis.

\begin{tabular}{|c|c|}
\hline Step & Action taken \\
\hline Reading the transcript & $\begin{array}{l}\text { Browse all transcripts } \rightarrow \text { make impression notes } \rightarrow \text { read transcripts one by one } \rightarrow \text { re-read } \\
\text { transcripts } \rightarrow \text { read transcripts line by line }\end{array}$ \\
\hline Labelling, coding & $\begin{array}{l}\text { Label words, sentences, or sections with relevant concepts, actions, similarities, and } \\
\text { differences }\end{array}$ \\
\hline Condensing, "grouping codes into categories or themes" & $\begin{array}{l}\text { Read all codes } \rightarrow \text { identify most important codes } \rightarrow \text { combine two or more codes and/or } \\
\text { create new codes } \rightarrow \text { condense long and thick descriptions into short meaningful ideas or } \\
\text { concepts } \rightarrow \text { organize codes with similar content into meaningful categories or themes }\end{array}$ \\
\hline Describing categories & $\begin{array}{l}\text { Examine categories and summarize the content of each category into descriptive text } \rightarrow \\
\text { decide which categories are most relevant to the study aim and objectives } \rightarrow \text { conceptualize } \\
\text { underlying patterns }\end{array}$ \\
\hline Organizing categories & $\begin{array}{l}\text { Organize categories to identify emerging concepts and persistent patterns of behavior and } \\
\text { beliefs among respondents } \rightarrow \text { describe connections between categories } \rightarrow \text { group similar } \\
\text { categories } \rightarrow \text { final categories or themes }\end{array}$ \\
\hline Looking for meaning & Inspect categories for meaningful text $\rightarrow$ interpret meaning $\rightarrow$ summarize findings \\
\hline Verifying & Check credibility of the analytical process by reviewing/discussing codes and final themes \\
\hline Reporting & Communicate the findings \\
\hline
\end{tabular}

Table 2. Tattersall's approach: Five W's to describe observational activities.

What - We observed five participants from the beginning of a sedation period until recovery. We focused the observations on how participants used capnography; how they responded to alarms; how they interacted around capnography whenever there was an alarm; and how and when they initiated an intervention based on capnography alarms. Anesthetists performed deep sedation (by Propofol + Fentanyl) and nurses administered moderate sedation (by Fentanyl + Midazolam) under supervision of the endoscopy physician.

- The anesthetist was present throughout the whole endoscopy procedure during deep sedation, but not during moderate sedation.

- Most performed endoscopies were colonoscopies and esophago-gastro duodenoscopies.

- Participants administered supplemental oxygen to all patients using a face mask or nasal cannula.

- Applied monitors included standard monitors (blood pressure, $\mathrm{ECG}^{*}$, and pulse oximeter) + capnography.

- All participants used capnography monitoring, either through the anesthesia machine or portable capnography device.

- Participants encountered complications with sedation such as, $\mathrm{SpO}_{2}<75^{* *}$, Bronchospasm, and Overshoot of sedation.

- Participants performed interventions in response to capnography such as, head tilt and chin lift, verbal/physical stimulation, bag-valve ventilation, nasal airway adjunct application, and procedure interruption.

Where We conducted observations in the endoscopy unit

When We conducted observations within a pre-determined timeframe, from 25 November 2018 through 30 December 2018.

Who Refer to Table 3

Why We aimed to explore practitioners' behavior while using capnography monitoring

*ECG, electrocardiogram; **S $\mathrm{Spo}_{2}$, percentage of oxygen saturation.

Table 3. Summary of participants' characteristics $(n=5)$.

\begin{tabular}{|c|c|c|c|c|c|}
\hline Demographic characteristic & Participant 1 & Participant 2 & Participant 3 & Participant 4 & Participant 5 \\
\hline Pseudonym & Martin & Joe & Alex & Sara & Mary \\
\hline Gender & Male & Male & Male & Female & Female \\
\hline Age & 48 & 36 & 56 & 34 & 27 \\
\hline Profession & \multicolumn{2}{|c|}{ Anesthetist Anesthesia technicia } & n Anesthetist & Sedation nurse & Sedation nurse \\
\hline Qualified (years) & 17 years & 13 years & 25 years & 12 years & 7 years \\
\hline Experience with endoscopy sedation (years) & 5 years & 10 years & 15 years & 9 years & 4 years \\
\hline Prior experience with capnography in other hospital settings & Yes & Yes & Yes & No & No \\
\hline
\end{tabular}




\section{Safety culture of endoscopy sedation}

It is important to explore sedation practice in order to understand how sedation practitioners utilize capnography during sedation. Within this theme, we captured a shared perception of the importance of safe sedation practice. During observations, participants used capnography as part of hospital policy during sedation. Participants shared similar views of what constituted safe sedation, considering hospital policy as a major element for safe sedation practice. Participants recognized the value of capnography to avoid respiratory complications and maintain adequate level of sedation. Martin stated: "Wherever sedation is administered, we use capnography and it's safe, but if capnography was not available, definitely there would have been complications".

Alex added: "Simply patient safety comes first, and we are simply following standards". Sara also stated that it was: "very rare [that] we have a complication".

One participant (Mary) believed that sedation complications were not serious in nature, and are easily managed: "I cannot consider them as serious complications. They are only the side-effects of medications we are giving, like Midazolam and Fentanyl". Mary believed that patient assessment is what ensured safe sedation: "actually sedation is safe as long as you assess the patient correctly". Observations of Mary were consistent with this statement, as she mainly relied on patient observation and assessment and only briefly glanced at capnography data. Ultimately, participants shared the notion that they delivered sedation safely in the endoscopy unit with the aid of what they perceived to be adequate monitoring practice.

\section{Professional identity and role in safe sedation practice}

Within this theme, we explored the different professional cultures of anesthesia practitioners and nurses in terms of values related to patient safety and responsibilities during sedation. Anesthesia practitioners were responsible for establishing a patient safety culture, while nurses were only expected to be a team player. Anesthesia practitioners established the patient safety initiatives for the whole sedation team. Martin said:

"Because of the efforts of the manager of off-site location anesthesia, we always work up-to-date during sedation regarding guidelines and equipment. We go according to any advances in patient safety in anesthesia".

\section{Alex also stated:}

"When sedation is performed by non-anesthetists, they have to consider all [of] the safety precautions for the patient when performing sedation. (...) As part of our sedation policy, capnography is a must, whether under anesthesia care or not under anesthesia care".
Nurses were not involved in establishing the policy for safe sedation. Mary said: "The anesthesia department is encouraging us to use the capnography monitoring when we are giving sedation".

Anesthesia practitioners perceived the notion of taking responsibility of patient safety as part of their professional identity, and they expressed their support to prepare endoscopy nurses to perform moderate sedation independently. Alex stated:

"Deep sedation is only performed by anesthetists, and we have two standard courses to prepare nonanesthesia physicians and nurses for [administering] moderate sedation, and they have to go through those two courses to work in sedation".

Anesthesia practitioners appreciated working alongside nurses; they believed that nurses contributed to patient safety and stressed the need to have competent nurses as members of the sedation team. Alex said:

"A couple of years ago, sedation was only given under anesthesia care, but for five years now, moderate sedation is given without supervision of anesthetists, and have not recorded major complications. We have key performance indicators that we are monitoring for complications: any aborted or admitted patient; any code blue; any airway obstruction, and we have no complication recorded up until now. (...) Sedation nurses - of course - have to go through a couple of courses before they are allowed to perform sedation and other courses to maintain being privileged. They are trained under anesthesia for ten to fifteen cases, and then they are left alone to perform sedation and to monitor their patients".

Nurses described their role in the sedation team as clear and well defined, and their responsibility was proportional to the training they possessed. Sara said:

"There is a policy for nurses to do sedation: One sedation nurse and a doctor, and to be a sedation nurse, we need to pass a few courses, and then they [anesthetists] will check all the actual given sedation cases under the supervision of anesthesia, and you will do the airway management course, and every two years you need to renew. We also have formal training to deal with the equipment".

Although the nurses felt they were trusted to maintain patient safety standards during sedation, they often did not use capnography adequately with sedated patients during observations. Nurses also expressed themselves to be self-sufficient professionals who demonstrate confidence 
in their work and abilities to perform moderate sedation and monitor their patients. Mary said:

"Hmmm if you are a sedation nurse and you are allowed to do sedation, I think you would have the knowledge to read capnography because it is included in our training: That's how we get privileged".

Despite the fact that nurses felt they were privileged, during observations, nurses did not seem like a full member of the team when asking questions and/or seeking help and were reluctant to express concerns with capnography when issues arose.

\section{Personal experience and knowledge about capnography performance}

\section{Value of capnography}

Participants stressed the value of past experience with the use of capnography to their ability to properly understand how capnography works during sedation. Participants linked past experience with capnography with the ease of use. Joe stated: "When I first started to work here, I didn't know much about capnography from my background". Martin added: "In my previous workplace, there was capnography as well in endoscopy, I used it for many years; capnography is basically simple to use".

One participant (Mary), who was a nurse with the least experience with capnography among all participants (four years) said: "I am really comfortable with capnography practice here". However, during observation, Mary did not seem comfortable with capnography when encountering technical issues, and required assistance from a more experienced nurse. Mary's statement suggests comfort with the policy of using capnography (following the rules) but not necessarily with how to use capnography. Sara, who was a more experienced nurse, generally seemed more comfortable with capnography than Mary during observations.

Participants also shared their experiences and opinions about capnography function, interpretation, and alarms. We captured several differences in the level of knowledge among participants. Anesthesia practitioners commonly believed having adequate knowledge and skills with the capnography monitoring was a core component for their professional identity. Joe stated:

"I believe it's really important for anyone who works in anesthesia to have good knowledge and good skills with capnography because it's [...] common equipment in our everyday job; to read capnography".

Alex, who was the most experienced participant, emphasized the value of capnography during sedation:
"We don't use capnography for the reading because it is constantly fluctuating, but rather we look for the waveform. The important thing is that we monitor that the patient is breathing despite the reading. We are monitoring that the patient has no upper airway obstruction and that the patient is ventilating well".

Anesthesia practitioners relied on the capnography waveform to assess their patients: The presence of a waveform gave them a sense of reassurance that the airway was secured.

Unlike anesthesia practitioners, nurses placed little emphasis on the waveform and monitored the numerical reading of capnography. Mary said:

"The readings are very helpful to assess my patient. Sometimes you can see the patient's oxygen saturation is 100 , but once you look at the patient, the patient is not really breathing! so we don't only rely on the pulse oximetry alone, capnography helps you to be comfortable and sure that your patient is breathing nicely. At least you won't panic if you can see [a] capnography reading while the patient is starting to desaturate".

While nurses acknowledged the reassurance capnography added to pulse oximetry and patient observation, and expressed confidence with their knowledge about capnography, they only used it as a secondary supportive monitor and relied mainly on patient observation and vital signs during observations to direct clinical judgement. Sara stated: "capnography helps a lot to support patients' vital signs".

\section{Capnography alarms}

Participants highlighted the concern that many false alarms limited capnography value. Participants exhibited different behaviors when responding to alarms during observation. Anesthesia practitioners identified the source of alarms and relied on these alarms to initiate an appropriate intervention if needed. Martin said:

"Sometimes the capnography gives false alarms. The patient is breathing but the capnography is showing a flat line. This is usually a technical error with the equipment and [there is] no need for any intervention".

Joe further indicated that it is necessary to have adequate skills to differentiate clinically relevant alarms from false alarms. He said:

"Sometimes we get confused with the alarms, but if you're able to interpret the capnography monitor to exclude artefacts, you should be fine". 
Nurses considered capnography alarms as disturbing. Sara said:

"Actually, capnography gives so many alarms. They are annoying because sometimes it's not an accurate alarm; only because the patient is moving, or the procedure interferes sometimes".

During observations, anesthesia practitioners responded to capnography alarms first by looking closely at the patient and constantly at the capnography screen, and then intervened accordingly; while nurses relied greatly on patient observation to initiate an intervention and tended to ignore most of the alarms, considering them false.

Nurses highlighted the lack of clear policy for capnography alarm management. Mary stated:

"I believe that the thing that disturbs me the most, the only thing I encounter, is the sound of alarm! Once the capnography is dropping, you know the sound of the alarm. And there is no clear policy, I think, to respond to capnography alarms".

Mary's statement is cause for concern, because it suggests that the sound of alarm disturbed Mary rather than worrying about the cause of the alarm. Her statement also reflected frustration with the lack of policy to differentiate true from false capnography alarms.

\section{Facilitators and barriers to the utilization of capnography monitoring}

Within this theme, participants shared their opinions of the factors that foster or discourage the adequate use of capnography. Figure 1 demonstrates these factors, which are condensed into three major categories of Sheckley et al.'s Modified Trio Model for Professional Learning $^{27}$ to successfully implement new technologies.

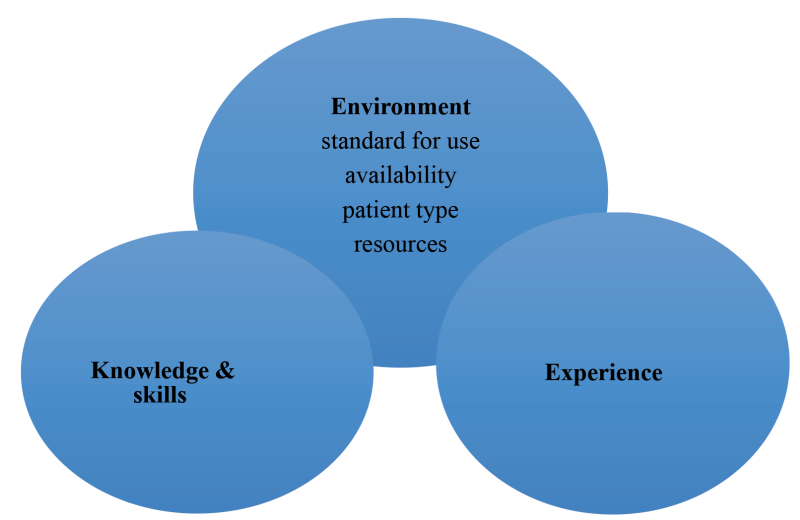

Figure 1. Modified Trio Model for Professional Learning by Sheckley et al.

\section{Environment}

Participants perceived ensuring patient safety as a strong facilitator for the use of capnography during sedation. Sara stated: "With capnography, I am more confident that the patient is breathing. I guess I can know my patient is safe". Alex further added that the fact that capnography is the standard of care promoted its use within the department. He said: "We are following standards; we are not inventing anything".

Participants perceived the availability and access to capnography equipment as important promoters for its use. Alex said that it was used:

"...either through the anesthesia machine or in some areas where no anesthesia machines were available, we managed to have a portable capnography [instrument], and we have different versions of portable capnography [instrument], as well".

However, Sara believed that the mere existence of the monitor was not sufficient for its utilization as long as it was associated with so many problems; she said: "Sometimes we can't use the machine, because there is some malfunction or need for recalibration".

Participants also believed that working in a major hospital with critically ill patients promoted the use of capnography. Alex stated:

"Here in this center, we have so many critical patients, and as a tertiary center, the majority of our patients and referral critical cases. We are dealing with very old, risky patients, and the procedures are long and complex. So, this makes us put efforts to make sure that the patient is safe and receives the best of care."

The cost burden of capnography represented a reasonable barrier to utilization. Joe said: "Not all hospitals have the resources to provide capnography". However, Martin stressed that capnography was cost-effective and should not be discouraged by cost, as he stated:

"If we are preventing complications from happening so we are safe. We are cost effective for the hospital in terms of discharging patients, instead of getting complications and re-admitting patients and spending more resources".

\section{Experience}

Participants believed that unfamiliarity with capnography monitoring equipment was a barrier to acceptance and adequate use by practitioners. Joe stated: "Lack of experience; make clinicians less confident around capnography. It distracts them from the patient because they do not understand how it works". Observations in the current study showed that practitioners with previous experience 
with capnography demonstrated a better pattern of utilization than those with fewer years of experience.

\section{Knowledge and skills}

Participants perceived knowledge and skills as important facilitators to adequate utilization. Mary said: "The clinician who is administering sedation and the one who is involved in the procedure must have the knowledge about capnography to be able to use it". During observations, anesthesia practitioners demonstrated superior capnography skills to sedation nurses.

\section{Discussion}

Within this study, we aimed to explore sedation practitioners' perceptions and behavior towards capnography during endoscopy sedation and examine how capnography monitoring influenced clinical decision-making when assessing ventilation. We also examined factors facilitating and barring the use of capnography. Our findings show that although participants perceived capnography as essential within the endoscopy unit and as part of hospital policy for sedation, the underlying beliefs which guided its utilization differed between anesthesia practitioners and nurses.

The safety of any culture is characterized by effective preventive measures and by practitioners' shared perceptions of the importance of safety. ${ }^{28}$ Capnography improves patient safety during sedation while serving as an early indicator of respiratory depression, which usually proceeds hypoxemia. ${ }^{6,7}$ Findings revealed a cultural difference between anesthesia practitioners and nurses in terms of how they monitored their sedated patients; while the former relied on technology, the latter relied on assessment with vital signs and patient observation.

Our findings show that anesthesia practitioners realized the value of capnography waveform to assess ventilation. Notably, the capnography waveform allows visual breath-by-breath assessment of ventilation, which is known as superior to the numerical reading when assessing ventilation during sedation. ${ }^{6,7}$ Anesthesia practitioners expressed a definite link between safety and the use of capnography, and thus, that its absence presented a potential risk to patients. However, nurses lacked the in-depth understanding of the value of capnography waveform during sedation. Nurses perceived the traditional method of patient observation and assessment of vital signs to be the best way to ensure adequate ventilation and patient safety. Clark et al. ${ }^{29}$ revealed similar findings: they stated that $77 \%$ of nurses believed that capnography compromised patient safety, as it would distract them from clinically monitoring their patients. We found that nurses, unlike anesthesia practitioners, were only accustomed to use capnography monitoring during sedation because the guidelines mandated its use in the department, and not be- cause nurses valued the benefit of capnography when assessing ventilation. Technology-based monitors such as capnography are only as good as the user interpreting the information they provide. ${ }^{30}$ In this context, adequate utilization of capnography relies on the practitioner's knowledge and skills.

In explaining the discrepancy between the two professional groups, it is important to consider the wide range of professional disciplines that work as teams in different departments and to understand the interaction between cultures that work together. Each professional culture guides its individuals to a particular way of thinking and behavior. ${ }^{31}$ The literature suggests that professional groups perceive the patient safety culture within a single unit differently based on their profession's differing values and norms. ${ }^{31,32}$

Anesthesia practitioners and nurses have different learning environments, which could explain the diversity in their cultures. The environment of the operating theatre is associated with high risks and time pressure; anesthetists are trained to become leaders and take responsibility for decision making. In contrast, nurses are trained to work as part of a team rather than independent decision-makers. ${ }^{33}$ The values of each profession create a communication barrier with the other, as these values are unspoken and might be difficult to perceive by the other. Anesthesia practitioners and nurses could look at the same thing, capnography, yet see different things, because they have different values related to patient safety. ${ }^{34}$

The patient safety culture within our study seems to fit into the Hierarchy model of the four types of culture in Cameron and Quinn's competing values framework, ${ }^{35}$ in which power, uniformity, policy, and formal rules drive individuals. ${ }^{36}$ The monitoring requirement for sedation within the hospital mandated the use of capnography. Nurses perceived capnography as a supportive monitor for clinical assessment despite their positive opinions about it - their use of capnography was policy- rather than value-driven. Although the incorporation of capnography into clinical practice through hospital policy might increase patterns of use, ${ }^{30}$ it did not ensure adequate utilization by the nurses in our study. Similarly, Iyer et al. ${ }^{37}$ and Langhan et al ${ }^{38}$ noticed that protocol-driven practice did not ensure adequate utilization of monitoring and lead practitioners to varied practice. They also found that practitioners with previous experience used capnography more efficiently that those with limited experience. ${ }^{38}$ Similarly, in our study, those who have had previous experience with capnography expressed a higher level of comfort while using it.

Anesthesia practitioners believed that patient safety during sedation was their professional obligation, which shows the autonomy of anesthesia practitioners within this setting. ${ }^{39}$ They established patient safety initiatives for the whole team and controlled the choice and involvement of sedation nurses. There is an ancient difference between 
anesthetists and nurses, including authority and responsibilities - the issue of power among physicians and nurses is well-known in literature. ${ }^{39,40}$ Anesthesia practitioners' discretion to involve nurses in developing sedation policies initiatives reflects the prevailing professional culture, owing to the special authority of the anesthesia profession over nursing. ${ }^{39}$

While nurses recognized support from anesthesia practitioners in their use of capnography, an admission of to having insufficient knowledge or skills seemed to be in contrast with the privilege that anesthesia practitioners granted nurses to perform moderate sedation without supervision. Nurses also expressed values that showed that they wanted to be responsible for patient safety. This responsibility was associated with anesthetists' delegation to nurses within that particular setting. Nurses maintained their values while monitoring sedated patients - with an emphasis on patient observation and assessment to make clinical decisions - which stems from the core value of their profession, such as human dignity and integrity. ${ }^{36}$ Similarly, Hall ${ }^{32}$ suggests that nurses respect and value the story of the patient and will not depend as heavily on objective data as physicians. Nurses in our study did not share the same values as anesthesia practitioners regarding what constituted patient safety culture.

It is important to consider that anesthesia practitioners have long experience with the use of capnography in the operating theatre, ${ }^{6}$ which explains their high levels of comfort with it, while nurses have only recently performed sedation within the institution as authorized by anesthetists. Thus, these nurses had little experience with capnography monitoring prior to work as sedation nurses. Langhan et al. ${ }^{11}$ reported that nurses with the least experience were more likely to perceive capnography as a sophisticated technology. According to Benner, ${ }^{41}$ novice practitioners rely on rules to guide their judgement because they have no prior experience with the situation they encounter.

Capnography false-alarms were frequent during this study, occurring when patients were mouth-breathing, or when the sampling line was dislocated. False-alarms can mislead practitioners by showing a flat line (indicating an apnea) while the patient is demonstrating adequate chest rise and able to maintain proper oxygenation. ${ }^{7,9} \mathrm{~A}$ study by Siebig et al. ${ }^{42}$ found that $40 \%$ of capnography alarms were false, and only $15 \%$ of all alarms were clinically important. Nurses' observed behavior in ignoring alarms suggests that they mainly trusted their own clinical assessment of the patient. However, the literature has identified this behavior as alarm fatigue (a tendency to ignore most alarms) ${ }^{43}$ and alarm fatigue leads clinicians to miss clinically important alarms in the future. Sowan et al. ${ }^{44}$ showed that a lack of alarm management policy regarding response to capnography alarms adds complexity to the recognition of important alarms.

The variation we observed in this study between anes- thesia practitioners and nurses highlight challenges when adopting new technologies. Nurses perceived a lack of familiarity with capnography to be a barrier for adequate use. Barnett et al. ${ }^{8}$ surveyed nurses to assess their use of capnography during sedation and found that most nurses were neutral or unsure when asked to rate how useful capnography was to the provision of safe sedation, and also neutral/unsure about the ease of interpreting capnography data. Langhan et al. ${ }^{11}$ revealed that only $20 \%$ of practitioners used capnography during sedation, and the reasons for not using it included lack of familiarity and lack of perceived need for its use. ${ }^{8}$ Maddox and Williams ${ }^{45}$ introduced capnography outside the operating theatre, and encountered similar challenges to those observed in this study, such as compliance with use and management of false alarms. These finding, combined with ours, provide insight into the importance of adequate training of practitioners on how to use and interpret capnography early in its implementation. Levels of knowledge can be measured by the use of appropriate instruments to help identify knowledge gaps and the need for further education. Kiekkas et al..$^{30}$ developed a comprehensive instrument to evaluate nurses' knowledge of capnography, which can increase nurses' knowledge and comfort levels with this technology.

Our study has several limitations. Notably, we conducted this study at a single endoscopy unit in a single hospital. The study participants have different levels of experience with capnography, and their behavior and perceptions of capnography might change after ongoing experience. We accept and acknowledge that our study sample is small and therefore is unlikely to represent the full spectrum, so the findings may not be transferable to other sedation practitioners. However, we managed this impact through triangulation of data collection methods to capture a wider perspective. We do not claim our findings to be generalizable to other communities, ${ }^{17}$ since the aim of focused ethnography is to understand, describe and interpret shared experiences and meanings within a particular cultural setting.

\section{Conclusions}

Although the literature has proven capnography monitoring to maintain patient safety by ensuring adequate ventilation during sedation, ${ }^{7-9}$ the practitioners' experiences and perceptions who use capnography have not yet been addressed. Within this focused ethnography, we explored the behavior and perceptions of five sedation practitioners ( 2 anesthetists, 1 anesthesia technician, and 2 nurses) regarding capnography monitoring during endoscopy sedation.

Our findings revealed varied behaviors and opinions about the value of capnography monitoring. While anesthesia practitioners relied heavily on capnography to assess ventilation, nurses paid more attention to patient 
observation and assessment. There was a prevailing culture rather than a shared professional culture between anesthesia practitioners and nurses: Anesthesia practitioners established the rules for safe sedation and monitoring requirements, and nurses were good team players who followed the rules. However, the unique cultures of each profession guided their perception of the value of capnography.

Our findings invite future research to explore-in more depth — what factors beyond professional identity, hospital rules and policy, and past experience are associated with the utilization of capnography during sedation. Our findings also point to the complexity of capnography alarms and the need for research to determine an appropriate alarm management protocols to guide practitioners.

Anesthesia practitioners advocated the patient safety initiatives in the endoscopy settings. Our findings give insight for future research to explore the notion of a cultural shift - to involve nurses in the implementation phase of capnography, and to establish shared values to what constitutes a patient safety culture.

\section{References}

1. Adams L, Butas S, Spurlock Jr D. Capnography (ETCO2), respiratory depression, and nursing interventions in moderately sedated adults undergoing transesophageal echocardiography (TEE). J Perianesth Nurs 2015;30:14-22.

2. Vaessen HH, Knape JT. Considerable variability of procedural sedation and analgesia practices for gastrointestinal endoscopic procedures in Europe. Clin Endosc 2016;49:47-55.

3. Joint Commission. Pain assessment and management standards for hospital: requirement, rationale, reference. a complimentary publication of the joint commission; 2017. Available from: https://www.jointcommission.org/assets /1/18/R3_Report_Issue_11_Pain_Assessment_8_25_17_FI NAL.pdf Accessed: December 02, 2018.

4. European Board of Anesthesiology. Recommendations for minimal monitoring during Anesthesia and Recovery; 2011. Available from: http://www.eba-uems.eu/resources/PDFS/ safety-guidelines/EBA-Minimal-monitor.pdf Accessed: April 20, 2018.

5. American Society of Anesthesiology. Standards for basic anesthetic monitoring: American Society of Anesthesiologists; 2010. Available from: https://www.asahq.org/standards-and-guidelines/standards-for-basic-anesthetic-monitor ing Accessed: April 27, 2018.

6 . Kodali BS. Capnography outside the operating rooms. Anesthesiol 2013;118:192-201.

7. Beitz A, Riphaus A, Meining A, et al. Capnographic monitoring reduces the incidence of arterial oxygen desaturation and hypoxemia during propofol sedation for colonoscopy: a randomized, controlled study (ColoCap Study). Am J Gastroenterol 2012;107:1205-12.

8. Barnett S, Hung A, Tsao R, et al. Capnographic monitoring of moderate sedation during low-risk screening colonoscopy does not improve safety or patient satisfaction: a prospective cohort study. Am J Gastroenterol 2016;111:388-94.

9. Klare P, Reiter J, Meining A, et al. Capnographic monitoring of midazolam and propofol sedation during ERCP: a randomized controlled study (EndoBreath Study). Endoscopy 2016;48:42-50.

10. Egan E. Introducing capnography monitoring into an endoscopy service. Gastroenterol Nurs 2016;14:37-43.

11. Langhan ML, Kurtz JC, Schaeffer P, et al. Experiences with capnography in acute care settings: a mixed-methods analysis of clinical staff. J Crit Care 2014;29:1035-40.

12. Brewer J. Ethnography. Philadelphia, PA: Open University Press; 2000.

13. Richards L, Morse JM. Readme first for a user's guide to qualitative methods. $3^{\text {rd }}$ ed. Thousand Oaks, CA: Sage; 2013.

14. Roper JM, Shapira J. Ethnography in nursing research. Thousand Oaks, CA: Sage; 2000.

15. Patton MQ. Qualitative research \& evaluation methods: Integrating theory and practice. $4^{\text {th }}$ edition. London: Sage; 2015.

16. Cooper J, Lewis R, Urquhart C. Using participant or nonparticipant observation to explain information behavior. Inf Res 2004;9:9-4.

17. Fetterman DM. Ethnography: Step-by-step. $3^{\text {rd }}$ ed. London: Sage Publications; 2009.

18. Bryman A. Social research methods. $5^{\text {th }}$ ed. Oxford: Oxford University Press; 2015.

19. Creswell JW, Creswell JD. Research design: Qualitative, quantitative, and mixed methods approaches. $5^{\text {th }}$ ed. Thousand Oaks, CA: Sage; 2018.

20. Renz SM, Carrington JM, Badger TA. Two strategies for qualitative content analysis: An intramethod approach to triangulation. Qual Health Res 2018;28:824-31.

21. Cardiff University. Research Integrity and Governance Code of Practice.; 2019. Available from: https://www.cardiff.ac.uk /_data/assets/pdf_file/0004/937021/Research-Integrityand-Governance-Code-of-Practice-v3-PDF.pdf Accessed: December 15, 2018.

22. Data Protection Act 1998, c. 29. B. Available from: http:// www.legislation.gov.uk/ukpga/1998/29/pdfs/ukpga_199800 29 en.pdf Accessed: December 20, 2018.

23. Ryan GW, Bernard HR. Techniques to identify themes. Field methods 2003;15:85-109.

24. Kvale S, Brinkmann S. Interviews: Learning the craft of qualitative research interviewing. $3^{\text {rd }}$ ed. Los Angeles: Sage Publications; 2015.

25. Carcary M. The research audit trial — enhancing trustworthiness in qualitative inquiry. J Bus Res 2009;1:7.

26. Tattersall A. Who, What, Where, When, Why: Using the 5 Ws to communicate your research. Impact of Social Sciences Blog 2015. Available from: http://eprints.lse.ac.uk/ 70619/1/blogs.1se.ac.uk-Who\%20What\%20Where\%20 When $\% 20$ Why\%20Using\%20the $\% 205 \% 20 \mathrm{Ws} \% 20$ to $\% 20$ c ommunicate\%20your\%20research.pdf Accessed: Jan 15, 2019

27. Sheckley B, Kehrhahn M, Bell S, Grenier R. Trio: an emerging model of adult professional learning. InProceedings of the 49th Annual Adult Education Research Conference 2008, University of Missouri, St. Louis. Available from: https://newprairiepress.org/aerc/2008/roundtables/12/ Accessed: Feb 02, 2019

28. Feng X, Bobay K, Weiss M. Patient safety culture in nursing: a dimensional concept analysis. J Adv Nurs 2008;63: 310-19.

29. Clark CL, Weavind LM, Nelson SE, et al. Nursing attitudes 
towards continuous capnographic monitoring of floor patients. BMJ Open Qual 2018;7:e000416.

30. Kiekkas P, Stefanopoulos N, Konstantinou E, et al. Development and psychometric evaluation of an instrument for the assessment of nurses' knowledge on capnography. Collegian 2016;23:39-46.

31. Danielsson M, Nilsen P, Rutberg H, Carlfjord S. The professional culture among physicians in Sweden: potential implications for patient safety. BMC Health Serv Res 2018;18:543.

32. Hall P. Interprofessional teamwork: Professional cultures as barriers. J Interprof Care 2005; 19:188-96.

33. Reese DJ, Sontag MA. Successful interprofessional collaboration on the hospice team. Health Soc Work 2001;26:16775.

34. Petrie HG. Do you see what I see? The epistemology of interdisciplinary inquiry. Educ Res 1976;5:9-15.

35. Cameron KS, Quinn RE. Diagnosing and changing organizational culture: Based on the competing values framework. $3^{\text {rd }}$ ed. San Francisco, CA: John Wiley \& Sons; 2011.

36. Jun J, Kovner CT, Dickson VV, et al. Does unit culture matter? The association between unit culture and the use of evidence-based practice among hospital nurses. Appl Nurs Res 2020;53:151251.

37. Iyer NS, Koziel JR, Langhan ML. A qualitative evaluation of capnography use in paediatric sedation: perceptions, practice and barriers J Clin Nurs 2015;24:2231-8.

38. Langhan ML, Shabanova V, Li FY, et al. A randomized controlled trial of capnography during sedation in a pediatric emergency setting. Am J Emerg Med 2015;33:25-30.

39. Makary MA, Sexton JB, Freischlag JA, et al. Operating room teamwork among physicians and nurses: teamwork in the eye of the beholder. J Am Coll Surg 2006;202:746-52.

40. Braaf S, Manias E, Finch S, et al. Healthcare service provider perceptions of organisational communication across the perioperative pathway: a questionnaire survey. $\mathrm{J}$ Clin Nurs 2013;22:180-91.

41. Benner P. From novice to expert. Am J Nurs 1982;82:402-7.

42. Siebig S, Kuhls S, Imhoff M, et al. Intensive care unit alarmsHow many do we need?. J Crit Care Med 2010;38: 451-56.

43. Applegate RL 2nd, Lenart J, Malkin M, et al. Advanced monitoring is associated with fewer alarm events during planned moderate procedure-related sedation: a 2-part pilot trial. Anesth Analg 2016;122:1070-78.

44. Sowan AK, Tarriela AF, Gomez TM, et al. Nurses' perceptions and practices toward clinical alarms in a transplant cardiac intensive care unit: Exploring key issues leading to alarm fatigue. JMIR Hum Factors 2015;2:e3.

45. Maddox RR, Williams CK, Fields M. Respiratory monitoring in patient-controlled analgesia. Am J Health Sys Pharm 2004;61:2628-35. 